\title{
Link Positions Matter: A Noncommutative Routing Metric for Wireless Mesh Networks
}

\author{
Gentian Jakllari ${ }^{\dagger}$, Stephan Eidenbenz*, Nicolas Hengartner*, Srikanth V. Krishnamurthy ${ }^{\dagger}$, Michalis Faloutsos ${ }^{\dagger}$ \\ $\dagger$ University of California, Riverside, *Los Alamos National Laboratory \\ Email: \{jakllari,krish,michalis\}@cs.ucr.edu, \{eidenben,nickh\}@lanl.gov
}

\begin{abstract}
We revisit the problem of computing the path with the minimum cost in terms of the expected number of link layer transmissions (including retransmissions) in wireless mesh networks. Unlike previous efforts, such as the popular ETX, we account for the fact that MAC protocols (including the IEEE 802.11 MAC) incorporate a finite number of transmission attempts per packet. This in turn leads to our key observation: the performance of a path depends not only on the number of the links on the path and the quality of its links, but also, on the relative positions of the links on the path. Based on this observation, we propose ETOP, a path metric that accurately captures the expected number of link layer transmissions required for reliable end-to-end packet delivery. We analytically compute ETOP, which is not trivial, since ETOP is a noncommutative function of the link success probabilities. Although ETOP is a more involved metric, we show that the problem of computing paths with the minimum ETOP cost can be solved by a greedy algorithm. We implement and evaluate a routing approach based on ETOP on a 25-node indoor mesh network. Our experiments show that the path selection with ETOP consistently results in superior TCP goodput (by over $50 \%$ in many cases) compared to path selection based on ETX. We also perform an in-depth analysis of the measurements to better understand why the paths selected by ETOP improve the TCP performance.
\end{abstract}

\section{Introduction}

Reducing the number of link layer retransmissions in a wireless mesh networks is critical for ensuring high overall throughput. This can be achieved by selecting routes with inherently reliable links. This has a two-fold effect. First, the throughput of the flows using these paths is higher. Second, the throughput of the network as a whole increases, since the fewer transmissions lead to lower network-wide contention.

In this paper, we revisit the problem of computing the path with the minimum cost in terms of the expected number of link layer transmissions in mesh networks. Note that when we discuss the path cost, the term transmissions includes also retransmissions of packets. The distinguishing aspect of our work is that we account for realistic retransmission strategies at the link layer in contrast to most previous studies. Specifically, recent efforts that have considered the problem [9], [5], [10], [12], make a major implicit or explicit assumption that there are an infinite number of retransmission attempts per packet

\footnotetext{
${ }^{0}$ Prepared partially through collaborative participation in the Communications and Networks Consortium sponsored by the U. S. Army Research Laboratory under the Collaborative Technology Alliance Program, Cooperative Agreement DAAD19-01-2-0011. The U.S. Government is authorized to reproduce and distribute reprints for Government purposes notwithstanding any copyright notation thereon. The work was also partially supported by the NSF CAREER grant 0237920.

LA-UR-07-7750.
}

at the link layer. This assumption, as we discuss latter, greatly simplifies the problem of computing the end-to-end path cost. However, in practice, there are a bounded number of retransmission attempts at the link layer. In this more realistic setting, routing strategies that are developed with the previous metrics may result in paths which incur a high number of expected retransmissions at the link layer.

The key observation that motivates this work is that the cost of a path depends not only on the number of links on the path and the quality of these links, but also on the relative positions of the links on the path. In more detail, one has to account for the possibility that a packet may be dropped at the link layer given the bounded number of retransmissions at that layer. Furthermore, with a reliable transport protocol, such a dropped packet will be retransmitted from the source. Thus, a packet drop close to the destination is expensive, since it induces retransmissions (in the subsequent transport layer retransmission attempt) on links that were successfully traversed prior to the drop.

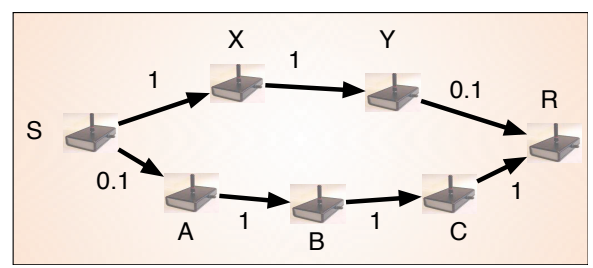

Fig. 1. The effect of the link positions on the performance of a path.

We illustrate this issue with the toy example in Figure 1. There are two paths from the source $\mathrm{S}$ to the destination $\mathrm{R}$; the number next to each link depicts the probability of a successful transmission (denoted as link success probability) across that link. At first glance, it may seem that it is better to use the path $[\mathrm{S}, \mathrm{X}, \mathrm{Y}, \mathrm{R}]$ instead of $[\mathrm{S}, \mathrm{A}, \mathrm{B}, \mathrm{C}, \mathrm{R}]$. In fact, previous strategies such as [9], will choose that path. However, the path $[\mathrm{S}, \mathrm{A}, \mathrm{B}, \mathrm{C}, \mathrm{R}]$ is better than $[\mathrm{S}, \mathrm{X}, \mathrm{Y}, \mathrm{R}]$. If the link layer performs at most two transmissions per packet (i.e., only one retransmission is allowed), it is easy to compute that the expected total number of link layer transmissions per packet is approximately 13 for the path $[\mathrm{S}, \mathrm{A}, \mathrm{B}, \mathrm{C}, \mathrm{R}]$, while it is approximately 20 for the path [S, X, Y, R]. The higher cost is due to the bad link that is closer to the destination, in the path $[\mathrm{S}, \mathrm{X}, \mathrm{Y}, \mathrm{R}]$.

Based on the aforementioned observation, we propose a path metric, which accurately captures the expected number of link layer transmissions assuming a finite number of retransmissions 
at this layer. We call our metric the Expected number of Transmissions On a Path or ETOP $P^{1}$ for short. ETOP considers the relative position of the links and thus, it is a noncommutative ${ }^{2}$ function of the link success probabilities unlike the previously proposed metrics. Our contributions can be summarized as follows:

(i) We derive a closed form expression to compute the ETOP cost of a path. Note that this derivation is non-trivial; the ETOP cost cannot be computed as a simple sum of link level metrics, because of the finite number of retransmissions at the link layer.

(ii) We prove that, despite its more involved calculation, ETOP satisfies: (a) the greedy-choice property, and (b) the optimal sub-structure property. Thus, computing the paths of minimum ETOP cost can be achieved with a greedy approach, and we develop an algorithm to that effect.

(iii) We develop and implement ETOP-R, an ETOP-based routing protocol. We evaluate its performance on an indoor wireless mesh network consisting of 25 nodes and compare it with that of ETX-based routing [9]. We observe that ETOP-R computes paths that yield higher TCP goodput (by over 50\% in many cases) compared to ETX-based routing.

(iv) We perform an analysis of the results at a microscopic level. With ETOP-R, we observe a reduced number of link layer transmissions and packet drops, and a higher TCP congestion window size (by as much as $300 \%$ ).

We wish to point out here that lossy links are fairly common in wireless mesh networks. This has been observed in prior work [9], [13] and we observe that losses due to failed MAC layer retransmissions are common in our indoor network. Note that we cannot simply blacklist and preclude the use of lossy links; the link quality fluctuates in time. Furthermore, some lossy links may be on exclusive paths to certain destinations.

The rest of the paper is organized as follows. In section II, we discuss related work. In section III, we analytically compute ETOP. In section IV, we show that the problem of minimizing the ETOP cost can be solved with a greedy algorithm. In section $\mathrm{V}$, we describe the implementation of ETOP-R. In section VI, we present our experimental results. Our conclusions form Section VII.

\section{Related Work and Background}

Many measurement studies [4], [25], [13], [22], [7], [20] have shown that links experience losses in wireless networks.

ETX: In [9], the authors design a link metric called ETX (for expected transmission count), which is equal to the inverse of a link's reliability. The end-to-end cost of a path is the sum of the ETX values of the links on the path; the routing layer simply computes routes that minimize this cost. A mechanism for estimating the link reliabilities, based on dedicated broadcast packets, was also proposed. Experiments on a 29-node 802.11 testbed showed that ETX-based routing results in better endto-end throughput as compared to minimum-hop routing [9].

\footnotetext{
${ }^{1} \mathrm{~A}$ preliminary summarized version of this work appears as a four-page extended abstract in MOBICOM 2007 [15].

${ }^{2} \mathrm{~A}$ function $f: A \times A \rightarrow B$ from a set $A$ to a set $B$ is said to be noncommutative if, $f(y, z) \neq f(z, y) \forall y, z \in A$.
}

Other Related Efforts: The efforts in [12], [23], [24], [18], [5], [6] have used the inverse of the link reliability (ETX) in combination with other parameters (such as the link bandwidth [12]) for improving routing performance in multihop wireless networks. In [12], Draves et al. propose a new routing metric, WCETT, that considers the link bandwidth and interference in addition to the (inverse of) the link reliability. Koksal et al. [6] propose mETX and ENT that extend ETX to account for highly variable link reliabilities. In [5], the authors use a metric similar to ETX for finding minimum energy paths. In a follow up effort [10], the authors introduce two more models, one where the link layer performs no retransmissions, and a second where the network either allows no link layer retransmissions, or the number of such retransmissions is estimated by the ETX metric. In [23], the product of ETX with the distance traversed towards the destination, is used for energy-efficient geographic routing. In [24] the authors use an ETX like metric for routing in sensor networks. In [21], a similar model is used for energy efficient routing. In this work, routing is jointly considered with power control, and in addition to the unicast case, the multicast case is also considered. In [26], [18], based on measurements, the authors argue that using broadcast packets to estimate the link reliability for data packets could lead to inaccuracies. Therefore, both efforts propose algorithms for data-driven link reliability estimation.

Previous metrics are not affected by the position of the link on a path: We wish to stress that, the inverse of the link reliability estimates the expected number of transmissions (including retransmissions), $\mathbb{E}$, needed to send a packet across a link, with the implicit assumption that an infinite number of retransmissions is allowed on the link. Therefore, the link layer never drops a packet. To elucidate this, let $p$ be the probability of a successful transmission across a link. Assuming that the outcomes of the transmission attempts on the link are independent and identically distributed, $\mathbb{E}$ can be computed as:

$$
\mathbb{E}=\sum_{j=1}^{\infty} j(1-p)^{j} p=\frac{1}{p}
$$

Since the link layer never drops a packet, there is never a need for a transport layer retransmission. This simplifies the calculation of the retransmissions needed for reliable packet delivery over a path; the number of retransmissions depends only on the link quality and not on their positions, i.e., the calculation is commutative.

In practice, however, there are a bounded number of link layer transmission attempts (as with 802.11) per packet and a reliable transport protocol will need to perform an end-toend retransmission to cope with link layer packet drops. In this case, as discussed with our toy example in Figure 1, the relative position of the links on a path becomes important when computing the cost of a path.

\section{Computing ETOP}

In this section, we present an analytical model for computing the ETOP cost of a path. In our model, unlike previous efforts, we account for the bounded number of retransmission attempts at the link layer (leading to possible packet drops at this layer). 
We then assume that a transport layer protocol (such as TCP) performs end-to-end retransmission attempts (e2e attempts) until the packet is finally delivered to the destination.

We make the following assumptions.

a. The probability of a successful transmission on a link does not change between retransmission attempts. In other words, the outcomes of successful link layer transmission attempts are independent and identically distributed $(I I D)^{3}$.

b. Implicitly, we assume that the power and bit-rate used for each transmission by a node does not change. If nodes are allowed to change their transmission properties, the probability of success will vary.

Network representation and notation: We model the wireless network as a directed graph $G(V, E, w)$, where $V$ is the set of nodes and $E$ the links. Every link $i \in E$ is assigned a weight $0<p_{i} \leq 1$, which represents the packet delivery probability over that link with a single transmission attempt.

Consider the problem of sending a packet from a source node $v_{0}$, to a destination node $v_{n}$, along a $n$-link path via nodes $v_{1}$, $v_{2} \ldots v_{n}$. The source, node $v_{0}$, initiates an e2e attempt. First, the packet is passed on to the link layer, which will transmit it to node $v_{1}$. If successfully received by node $v_{1}$, it will then be transmitted to node $v_{2}$, and so forth, until the packet reaches node $v_{n}$. There is a probability $0<p_{i} \leq 1, i=1, \ldots, n$ that the packet, when transmitted by node $v_{i-1}$, will reach node $v_{i}$. If the packet transmitted by node $v_{i-1}$ does not reach node $v_{i}$, it is transmitted again by the link layer of node $v_{i-1}$. Up to $K$ transmission attempts (including the initial attempt) are made, and the packet is dropped if the $K^{\text {th }}$ transmission fails to reach node $v_{i}$. The drop is reported to the transport layer of node $v_{0}$. In response, the transport layer of $v_{0}$ initiates a new e2e attempt for the same packet. For every e2e attempt, there is a cost: the number of link level transmissions during this attempt. Let $T_{n}$ be a random variable that represents the sum of the costs of all the e2e attempts made in order for a packet to be delivered from node $v_{0}$ to node $v_{n}$. Our goal is to compute the expected value of $T_{n}$, the ETOP cost of the path, as a function of link weights, $p_{i}$, and the bound on the number of link level transmissions, $K$.

Let $Y_{n}$ denote the random variable representing the number of e2e attempts required in order for the packet to be delivered to the destination on the $n$-hop path. Let $M_{\ell}$ denote the number of consecutive hops that are successfully traversed along the path, beginning at node $v_{0}$, in the $\ell^{\text {th }} \mathrm{e} 2 \mathrm{e}$ attempt. Thus, $M_{\ell}=0$ if the packet fails to reach node $v_{1}$ from node $v_{0}$, and $M_{\ell}=n$ if the message has reached $v_{n}$. If $M_{\ell}<n$, the $(l+1)^{\text {st }}$ e2e attempt begins. We assume that the random variables $M_{1}, M_{2}, \ldots$ are independent and identically distributed (IID) and can be represented by a single random variable $M$. This implies that the effects experienced on the different e2e attempts are independent and identical. Since one might expect the fading to be a short-term effect [17], this assumption is reasonable. Let $H_{\ell, j}$ denote the number of link layer transmissions needed to deliver the packet from node $v_{j}$ to node $v_{j+1}$ in the $\ell^{\text {th }} \mathrm{e} 2 \mathrm{e}$

\footnotetext{
${ }^{3}$ This assumption is made to keep the computation tractable; a similar assumption is made in almost all related work (for example, in [9], [11]). Our experimentations on a real mesh network show that there are benefits with our approach in spite of this assumption.
}

attempt. If the message has successfully traversed the link from $v_{j}$ to $v_{j+1}, H_{\ell, j} \leq K$; else, if the message fails to reach node $v_{j+1}$ from node $v_{j}$, then, $H_{\ell, j}=K$ and a new e2e attempt is started at node $v_{0}$. For each node $v_{j}$, we assume that $H_{1, j}, H_{2, j}, \ldots$, are IID random variables and we use the notation $H_{j}$ to represent this common random variable.

To elucidate the meaning of the variables defined so far, we consider a simple scenario that can occur when a packet is transmitted from $v_{0}$ to $v_{4}$. Let there be two e2e attempts ( $\left.Y_{4}=2\right)$ to deliver a single packet from the node $v_{0}$ to node $v_{4}$. On the first e2e attempt, the packet crosses links $\left(v_{0}, v_{1}\right)$ and $\left(v_{1}, v_{2}\right)$ after being transmitted only once. However, it is dropped at node $v_{2}$. Therefore, $H_{1,0}=H_{1,1}=1, H_{1,2}=K$ and $M_{1}=2$. The cost in terms of link level transmissions incurred on this e2e attempt is $K+2$. On the second attempt, the packet is delivered to the destination, node $v_{4}$, and crosses each link with a single link layer transmission attempt. Therefore, $H_{2,0}=H_{2,1}=H_{2,2}=H_{2,3}=1$, and $M_{2}=4$. The cost in terms of link level transmissions incurred on this e2e attempt is 4 . The total cost incurred in terms of link level transmissions to deliver the packet from node $v_{0}$ to node $v_{4}$, is $T_{4}=K+6$.

The cost of a path: Using the model and the random variables defined above, for the general case of a $n$-link path, the cost, $T_{n}$, is given by:

$$
T_{n}=\sum_{\ell=1}^{Y_{n}}\left(\left[\sum_{j=0}^{M_{\ell}-1} H_{\ell, j}\right]+K \mathbb{I}\left(\ell<Y_{n}\right)\right)
$$

where, $\sum_{j=0}^{-1}=0$ and $\mathbb{I}\left(\ell<Y_{n}\right)$ represents the indicator function that takes on a value 1 when $\ell<Y_{n}$ and 0 otherwise. If $\ell<Y_{n}$, the specific e2e attempt failed to deliver the packet to the destination, i.e., the packet was dropped somewhere along the path. We know that the node at which the packet was dropped performed exactly $\mathrm{K}$ transmissions. The summation inside the parentheses simply represents the number of link level transmissions in the process of crossing $M_{\ell}$ links during the $\ell^{\text {th }}$ e2e attempt.

Theorem 1. The expected number of transmissions for delivering a packet over a path $\left(v_{0}, \ldots v_{n}\right)$, ETOP, is:

$$
\begin{aligned}
\mathbb{E}\left[T_{n}\right] & =\left(K+\sum_{j=0}^{n-2}\left(\mathbb{E}\left[H_{j} \mid H_{j} \leq K\right] \mathbb{P}[M>j \mid M<n]\right)\right) \\
& \times \quad \mathbb{E}\left[Y_{n}-1\right]+\sum_{j=0}^{n-1} \mathbb{E}\left[H_{j} \mid H_{j} \leq K\right] .
\end{aligned}
$$

Proof: To prove the claim, we first condition on $Y_{n}$, the number of e2e attempts. Then,

$$
\begin{aligned}
\mathbb{E}\left[T_{n}\right] & =\mathbb{E}_{Y_{n}}\left[\mathbb{E}\left[T_{n} \mid Y_{n}\right]\right] \\
& =\mathbb{E}_{Y_{n}}\left[\sum_{\ell=1}^{Y_{n}} \mathbb{E}\left[\sum_{j=0}^{M_{\ell}-1} H_{\ell, j} \mid Y_{n}\right]\right]+K \mathbb{E}\left[\sum_{\ell=1}^{Y_{n}} \mathbb{I}\left(\ell<Y_{n}\right)\right] \\
& =\mathbb{E}_{Y_{n}}\left[\sum_{\ell=1}^{Y_{n}} \sum_{j=0}^{n-2} \mathbb{E}\left[\mathbb{I}\left(M_{\ell}>j\right) H_{\ell, j} \mid Y_{n}\right]\right]+K \mathbb{E}\left[\left(Y_{n}-1\right)\right]
\end{aligned}
$$

The conditional expectation within the summation can be written as (by further conditioning on $M_{\ell}$ ):

$\mathbb{E}\left[\mathbb{I}\left(M_{\ell}>j\right) H_{\ell, j} \mid Y_{n}\right]=\mathbb{E}_{M_{\ell} \mid Y_{n}}\left[\mathbb{I}\left(M_{\ell}>j\right) \mathbb{E}\left[H_{\ell, j} \mid Y_{n}, M_{\ell}\right] \mid Y_{n}\right]$. 
Let us consider the case where a link $j$ is successfully traversed during the e2e attempt $l$, i.e., $j<M_{\ell}$ and $\ell \leq Y_{n}$. In this case the number of attempts on link $j$ can be at most $K$, i.e., $H_{\ell, j} \leq K$ (Note that if there is a failure on a link $j$, then $H_{\ell, j}=K$ and $M_{\ell}=j$.).

$$
\mathbb{E}\left[H_{\ell, j} \mid Y_{n}, M_{\ell}\right]=\mathbb{E}\left[H_{\ell, j} \mid H_{\ell, j} \leq K\right] \times \mathbb{I}\left(\ell \leq Y_{n}, j<M_{\ell}\right) .
$$

Since by definition, the $Y_{n}^{t h}$ attempt is the first time that $M_{\ell}=n$, it follows that $M_{\ell}<n$ for $\ell<Y_{n}$ and $M_{\ell}=n$ for $\ell=Y_{n}$. Hence for $j=0,1,2 \ldots, n-1$,

$$
\mathbb{E}\left[\mathbb{I}\left[M_{\ell}>j\right] \mid Y_{n}\right]=\left\{\begin{array}{cc}
\mathbb{E}\left[\mathbb{I}\left[M_{\ell}>j\right] \mid M_{\ell}<n\right] & \ell<Y_{n} \\
1 & \ell=Y_{n}
\end{array}\right.
$$

Substitution of the above expressions in (4) and recognizing that $\mathbb{E}\left[\mathbb{I}\left[M_{\ell}>j\right] \mid M_{\ell}<n\right]=\mathbb{P}\left[M_{\ell}>j \mid M_{\ell}<n\right]$ leads to:

$$
\begin{aligned}
\mathbb{E}\left[H_{\ell, j} \mid Y_{n}, M_{\ell}\right] & =\mathbb{E}\left[H_{\ell, j} \mid H_{\ell, j} \leq K\right] \mathbb{P}\left[M_{\ell}>j \mid M_{\ell}<n\right] \mathbb{I}\left[Y_{n}>\ell\right] \\
& +\mathbb{E}\left[H_{\ell, j} \mid H_{\ell, j} \leq K\right] \mathbb{I}\left[Y_{n}=\ell\right] .
\end{aligned}
$$

Summing the latter over $j \in\{0,1, \ldots, n-1\}$ and $\ell \leq Y_{n}$ and using the fact that $M_{\ell}$ are independent copies of $M$ and $H_{\ell, j}$ are independent copies of $H_{j}$, we get

$$
\begin{aligned}
\mathbb{E}\left[T_{n}\right] & =\mathbb{E}\left[\left(Y_{n}-1\right)\right] \sum_{j=0}^{n-2}\left(\mathbb{E}\left[H_{j} \mid H_{j} \leq K\right] \mathbb{P}[M>j \mid M<n]\right) \\
& +\sum_{j=0}^{n-1} \mathbb{E}\left[H_{j} \mid H_{j} \leq K\right]+K \mathbb{E}\left[\left(Y_{n}-1\right)\right] .
\end{aligned}
$$

which is the claimed result.

Next, we transform Equation (3) so that it can be expressed in terms of the link weights $\left\{p_{i}, i=1, \ldots n\right\}$, the bound on the number of link layer transmissions, $K$, and the length of the path, $n$.

Let $\pi_{i}, i=1, . ., n$ be the probability that the packet is not dropped on the link $\left(v_{i-1}, v_{i}\right)$. Given our assumptions, and since there will be at most $K$ link layer transmissions, $\pi_{i}=1-(1-$ $\left.p_{i}\right)^{K}$. For $i=1, \ldots$ define $\rho_{i}=\pi_{1} \times \cdots \times \pi_{i}$. The tail probability of $M$ is $\mathbb{P}[M>i]=\pi_{1} \times \pi_{2} \times \cdots \times \pi_{i+1}=\rho_{i+1} . Y_{n}$ has a geometric distribution with parameter $\mathbb{P}[M \geq n]=\pi_{1} \pi_{2} \times \cdots \times \pi_{n}=\rho_{n}$; this is because $Y_{n}=\ell$ implies that there are $\ell-1 \mathrm{e} 2 \mathrm{e}$ attempts that fail, followed by the e2e attempt that succeeds. It follows that

$$
\begin{aligned}
\mathbb{P}[M>i \mid M<n] & =\frac{\mathbb{P}[i<M<n]}{\mathbb{P}[M<n]}+\frac{\mathbb{P}[M>i]-\mathbb{P}[M \geq n]}{1-\mathbb{P}[M \geq n]} \\
& =\frac{\rho_{i+1}-\rho_{n}}{1-\rho_{n}} .
\end{aligned}
$$

Corollary 1. The expected cost of delivering a packet over a path $\left(v_{0}, \ldots v_{n}\right)$, the ETOP cost of the path, can be expressed simply in terms of the link weights $\left\{p_{i}, i=1, \ldots n\right\}$, the bound on the number of link layer transmissions, $K$, and the length of the path, $n$, as:

$$
\mathbb{E}\left[T_{n}\right]=\sum_{i=0}^{n-2}\left(E_{i} \frac{\rho_{i+1}}{\rho_{n}}\right)+K \frac{1-\rho_{n}}{\rho_{n}}+E_{n-1} .
$$

where $\rho_{i}=\pi_{1} \times \cdots \times \pi_{i}$ and $E_{i}=\mathbb{E}\left[H_{i} \mid H_{i} \leq K\right]$
Proof: Using Equation (5) and the newly defined variables, Equation (3) reduces to :

$$
\begin{aligned}
\mathbb{E}\left[T_{n}\right] & =\left(\frac{1}{\rho_{n}}-1\right)\left(K+\sum_{i=0}^{n-2} E_{i} \frac{\rho_{i+1}-\rho_{n}}{1-\rho_{n}}\right)+\sum_{i=0}^{n-1} E_{i} \\
& =\sum_{i=0}^{n-2} E_{i}\left(\frac{\rho_{i+1}-\rho_{n}}{\rho_{n}}+1\right)+K \frac{1-\rho_{n}}{\rho_{n}}+E_{n-1} \\
& =\sum_{i=0}^{n-2}\left(E_{i} \frac{\rho_{i+1}}{\rho_{n}}\right)+K \frac{1-\rho_{n}}{\rho_{n}}+E_{n-1} .
\end{aligned}
$$

We show that $E_{i}=\mathbb{E}\left[H_{i} \mid H_{i} \leq K\right]$, the expected number of transmission from node $v_{i-1}$ to $v_{i}$ can be computed simply as function of $p_{i}$ and $K$ in Lemma 1.

Lemma 1. The expected number of transmissions on a link $i \in E$ with weight $p$, for delivering a packet to the receiver, given that the number of attempts is bounded by a constant $K$ is:

$$
E\left[H_{i} \mid H_{i} \leq K\right]=\sum_{j=1}^{K} j \frac{(1-p)^{j-1} p}{1-(1-p)^{K+1}}
$$

Proof: $E\left[H_{i} \mid H_{i} \leq K\right]=\sum_{j=1}^{K} j P\left(H_{i}=j \mid H_{i} \leq K\right) \quad$ where,

$$
\begin{aligned}
\left.P\left(H_{i}=j \mid H_{i} \leq K\right]\right) & =\frac{P\left(H_{i}=j \cap H_{i} \leq K\right)}{P\left(H_{i} \leq K\right)} \\
& =\frac{P\left(H_{i}=j\right)}{1-P\left(H_{i}>K\right)}=\frac{(1-p)^{j-1} p}{1-(1-p)^{K+1}}
\end{aligned}
$$

Lemma 2. The ETOP metric is a noncommutative function of the link success probabilities.

Proof: Assume to the contrary that ETOP is a commutative function of the link success probabilities. If this is the case, the cost assigned by ETOP to the paths with link success probabilities $(1,1,0.2)$ and $(0.2,1,1)$ should be the same. Using Equation (6) and setting $K=3$, the ETOP costs for the two paths are 7 and 8.54, respectively. This contradicts our assumption and thus, ETOP is noncommutative.

Note that Equation (6) defines the ETOP metric. For every $n$, Equation (6) maps a $n$-link path to its ETOP cost. This mapping is not a simple sum of the link metrics, and is noncommutative. In Section IV, we design a simple greedy algorithms that can find minimum ETOP cost paths from a node to all the other nodes in the network.

\section{Our Greedy Routing Approach}

In this section, we first show that the problem of finding the path with the minimum ETOP cost between two nodes (minimum ETOP cost problem) can be solved by a greedy approach. Then, we present one such greedy algorithm.

Theorem 2. The minimum ETOP cost problem can be optimally solved by a greedy algorithm.

Proof: An optimization problem can be solved by a greedy algorithm, if two properties hold [8]: the greedy choice property, and the optimal sub-structure property. Thus, the proof is immediate from Lemmas 3 and 4. 
Lemma 3. The minimum ETOP cost problem satisfies the greedy-choice property.

Proof: It suffices to show that the cost of a path $X_{n+1}=$ $\left[v_{0}, \ldots, v_{n+1}\right]$ can be computed by: (a) the cost of the sub-path $X_{n}=\left[v_{0}, \ldots, v_{n}\right]$ and, (b) the weight of link $\left(v_{n}, v_{n+1}\right)$.

Let $\mathbb{E}\left[T_{n}\right]$ and $\mathbb{E}\left[T_{n+1}\right]$ be the cost of the above paths $X_{n+1}$ and $X_{n}$, respectively. We begin by using Equation (6) for $X_{n+1}$, and then, we express $\mathbb{E}\left[T_{n+1}\right]$ in terms of $\mathbb{E}\left[T_{n}\right]$ as follows:

$$
\begin{aligned}
\mathbb{E}\left[T_{n+1}\right]= & \sum_{i=0}^{n-1}\left(E_{i} \frac{\rho_{i+1}}{\rho_{n+1}}\right)+K \frac{1-\rho_{n+1}}{\rho_{n+1}}+E_{n} \\
= & \frac{1}{\pi_{n+1}} \sum_{i=0}^{n-2}\left(E_{i} \frac{\rho_{i+1}}{\rho_{n}}\right)+E_{n-1} \frac{1}{\pi_{n+1}}+K \frac{1-\rho_{n}}{\rho_{n}} \frac{1}{\pi_{n+1}} \\
- & K \frac{1-\rho_{n}}{\rho_{n}} \frac{1}{\pi_{n+1}}+K \frac{\left(1-\rho_{n} \pi_{n+1}\right)}{\rho_{n} \pi_{n+1}}+E_{n} \\
= & \frac{1}{\pi_{n+1}}\left[\sum_{i=0}^{n-2}\left(E_{i} \frac{\rho_{i+1}}{\rho_{n}}\right)+K \frac{1-\rho_{n}}{\rho_{n}}+E_{n-1}\right] \\
& +K \frac{\left(\rho_{n}-\rho_{n} \pi_{n+1}\right)}{\rho_{n} \pi_{n+1}}+E_{n} \\
= & \frac{1}{\pi_{n+1}} \mathbb{E}\left[T_{n}\right]+K \frac{1-\pi_{n+1}}{\pi_{n+1}}+E_{n}
\end{aligned}
$$

Recall that $\pi_{n+1}=1-\left(1-p_{n+1}\right)^{K}$ is the probability of no drop on the link $\left(v_{n}, v_{n+1}\right), p_{n+1}$ is the weight of the link $\left(v_{n}, v_{n+1}\right)$, $K$ is the bound on the number of retransmissions, and $E_{n}$ is the expected number of transmissions on that link.

Equation (7) shows that the cost of $X_{n+1}$ can be calculated by the cost of $X_{n}$ and the weight of link $\left(v_{n}, v_{n+1}\right)$, and this concludes the proof.

Lemma 4. The minimum ETOP cost problem satisfies the optimal sub-structure property, that is, the sub-path of an optimal path is itself an optimal path.

Proof: We prove the lemma by contradiction. Let us assume that the "minimum cost path" is $X_{m}=\left[v_{0}, \ldots, v_{m}\right]$ and assume that there exists at least one node such that the sub-structure property does not hold. Then, we can find a node $v_{n-1}$ such that: $X_{n-1}=\left[v_{0}, \ldots, v_{n-1}\right]$ in not optimal, while $X_{n}$ $=\left[v_{0}, \ldots, v_{n}\right]$ is optimal.

Using Equation (7), the cost of path $X_{n}$ is given below:

$$
\mathbb{E}\left[T_{n}\right]=\frac{1}{\pi_{n}} \mathbb{E}\left[T_{n-1}\right]+K \frac{1-\pi_{n}}{\pi_{n}}+E_{n-1}
$$

where, $E\left[T_{n-1}\right]$ is the cost of the path $X_{n-1}$.

Since we assumed that the sub-structure property does not hold for path $X_{n-1}$, there exists a path $X_{n-1}^{\prime}$ from $v_{o}$ to $v_{n-1}$, with cost:

$$
E\left[T_{n-1}^{\prime}\right]<E\left[T_{n-1}\right]
$$

Consequently, using path $X_{n-1}^{\prime}$, we can define another path $X_{n}^{\prime}$ from $v_{0}$ to $v_{n}$ with cost:

$$
\mathbb{E}\left[T_{n}^{\prime}\right]=\frac{1}{\pi_{n}} \mathbb{E}\left[T_{n-1}^{\prime}\right]+K \frac{1-\pi_{n}}{\pi_{n}}+E_{n-1}
$$

The terms $\frac{1}{\pi_{n}}, K \frac{1-\pi_{n}}{\pi_{n}}$ and $E_{n-1}$ are all positive. Thus, combining Equations (8), and (10) with Inequality (9), we conclude that: $\mathbb{E}\left[T_{n}^{\prime}\right]<\mathbb{E}\left[T_{n}\right]$. However, this contradicts the assumption that $\mathbb{E}\left[T_{n}\right]$ is the minimum cost from $v_{0}$ to $v_{n}$.
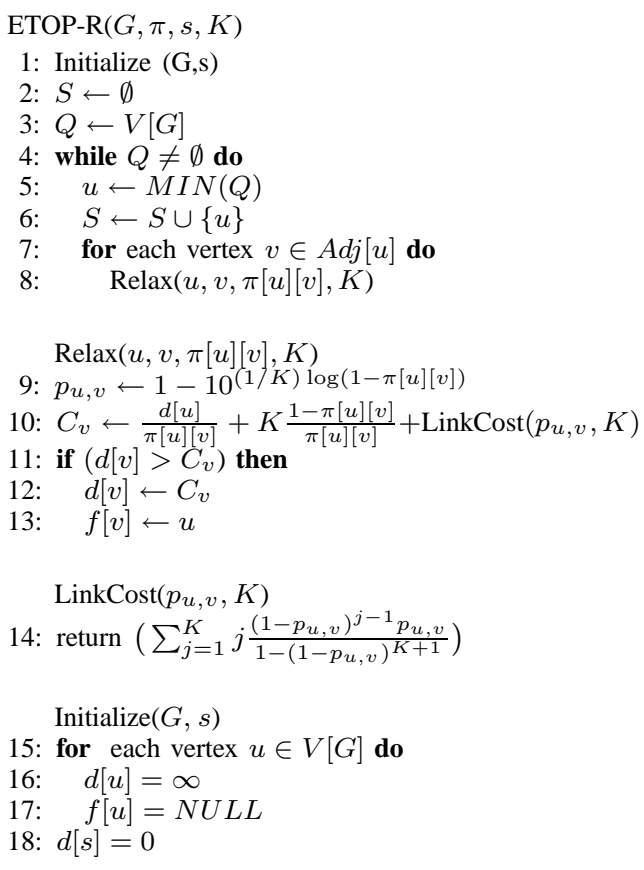

Fig. 2. Our ETOP-based algorithm for identifying the minimum ETOP cost path. It takes as input a graph $G(V, E)$, a source node $s$, the bound on the number of retries, $K$, and the $|V| \times|V|$ array of link probabilities of no drop, $\pi$.

\section{A. The ETOP-based algorithm (ETOP-R)}

Having proved that a greedy approach can compute the paths with the minimum ETOP cost, we design an algorithm based on Dijkstra's single-source shortest path algorithm [8] for doing so. The algorithm takes as input: (a) a graph representing the the network, (b) the edge weights, represented by the $\pi_{i}$ (the probability of no drop), (c) a bound on the number of retransmissions at the link layer, $K$, and (d) a source node. The algorithm computes the minimum ETOP paths from the source to every other node.

With Dijkstra's algorithm, if node $u$ immediately follows node $v$, on a path from the source node to $v$, and $d[u]$ is the cost of the path to $u$, the cost to $v$ is:

$$
d[v]=d[u]+w(u, v)
$$

where, $w(u, v)$ is the non-negative weight of link $(u, v)$.

In our case, the key observation is that with ETOP, from Equation 7, the cost to node $v$ is given by:

$$
d[v]=\frac{d[u]}{\pi[u][v]}+K \frac{1-\pi[u][v]}{\pi[u][v]}+\text { LinkCost }_{u, v}
$$

where, $\pi[u][v]$ is the probability of no drop between nodes $u$ and $v$, and LinkCost is the expected number of transmissions (including retransmissions) over link $(u, v)$ as defined in Lemma 1. Note that the cost functions in Equations 11 and 12 define optimization problems that satisfy the greedy-choice and sub-structure property. Based on this, we design ETOP-based routing, which is depicted in detail in Figure 2. The procedure differs from the Dijkstra's algorithm in the Relax procedure on line 10, where we replace Equation 11 with Equation 12. The correctness of our algorithm follows from the correctness of Dijkstra's algorithm. 


\section{Implementation}

In order to quantify the benefits of using ETOP, we implement a routing strategy based on the algorithm described in Section IV on a 25 node indoor wireless mesh network.

Our Experimental Network: Our indoor wireless mesh network (Figure 5) consists of 25 Soekris net4826 nodes, deployed on one floor our building. Each node runs a Debian v3.1 Linux distribution with kernel version 2.6. We have equipped nodes with EMP-8602-6G 802.11a/b/g WiFi cards [1], which embed the Atheros AR5006 chipset; the cards are controlled by the latest Linux MadWifi driver [3]. Each card is connected to a 5-dBi gain, external omnidirectional antenna. We use the $802.11 \mathrm{a}$ mode to avoid interference from co-located $802.11 \mathrm{~b} / \mathrm{g}$ networks. We use the popular Click toolkit [19] to implement our routing strategy.

Routing Implementation: We implement ETOP-based routing as part of a modified version of the dynamic source routing protocol (DSR) [16] developed by De Couto et al [9] for the Linux kernel. We chose DSR because (i) it is one of the most popular protocols for multihop wireless networks and hence, its implementations are readily available and (ii) it allows a source to decide on the path to the destination (required by ETOP-R since it is noncommutative). Furthermore, we consider the ETX metric for comparison and use the implementation of the routing strategy based on ETX [9]. For ease of notation we refer to ETOP-based routing as ETOP-R and to ETX-based routing as ETX-R.

DSR mechanics: With DSR [16], a node attempts to find a route to a destination by broadcasting a route request message (RREQ). The RREQ is subsequently re-broadcasted once by each nodes in the network, upon receipt. A node inserts its own address in the RREQ before re-broadcasting it. The sequence of addresses in the forwarded RREQ specifies the route traversed from the source to the destination. Upon receiving a RREQ, the destination sends a route reply message (RREP) to the source (with the route embedded within), along the reverse route recorded in the corresponding RREQ. The source stores the routes collected from all the RREPs received in a cache and uses, for a limited time, the route with the minimum hop count for forwarding data ${ }^{4}$. Note that as in [9], the route error messages (RERR messages) induced by DSR are disabled during the experiments; this functionality of DSR is not utilized with either ETX-R or ETOP-R.

Implementation of ETX-R: To estimate and use the link qualities, a link-measurement component was implemented by [9] as a separate element in Click [19]; it runs on every node and uses small broadcast packets to estimate the delivery probability from this node to each of its neighbors. The delivery probabilities computed by the link-measurement component are used to compute the ETX metric as described in Section II. With ETX-R, when a node forwards a RREQ it includes the ETX metric on the link to the node from which it received the RREQ. This information is then reported back to the source

\footnotetext{
${ }^{4} \mathrm{~A}$ complete description of the DSR protocol is omitted due to space limitations; instead, we briefly describe the route discovery mechanism because of its relevance to the ETX-R and ETOP-R implementations.
}

through the RREPs. At the source, this information is passed on to the link-measurement component, which maintains a cache (the ETX link cache) of all the known nodes and the ETX metrics of their corresponding links. Whenever the source needs a route, if the destination is in the cache, the linkmetric component will return the route with the minimum ETXweight, computed by running Dijkstra's weighted shortest path algorithm on the topology constructed with the nodes and links in the ETX link cache.

Implementation of ETOP-R To implement our ETOP-based algorithm, first we build a new cache for ETOP that is similar to the ETX link cache, except that the links are now represented by their delivery probabilities. The ETOP cache is not populated by collecting data via an explicit new mechanism, but is derived from the information in the ETX link cache. We exploit the simple relation between ETX and the link delivery probability $\left(E T X=\frac{1}{p_{\text {success }}}\right)$. In computing ETX, the authors assume that the probes compute the probability of successfully delivering a packet across a link; ETX is computed to be the inverse of this probability. With ETOP, the probability of successfully delivering a packet across a link is given by $\pi_{i}$, the probability that a packet is "not" dropped on a given link. Thus, we equate $\pi_{i}$ to $1 / E T X$. This value is then used in computing the ETOP cost as discussed earlier. Finally, the ETOP-based algorithm, described in Figure 2, is implemented within the link-measurement component; it takes as input the ETOP link cache and returns the route with the minimum ETOP cost.

Mapping our models to the implementation: The models (from Section III) for computing ETOP were based on a set of assumptions that the link delivery probabilities are IID. However, in reality, this may not always hold. Packet drops may sometimes reflect correlated behaviors. First note that it is extremely difficult to accurately characterize these correlations; second, it may also be difficult to take them into account while performing routing since they may have high temporal fluctuations. Thus, the goal of our design is to reduce the number of link layer retransmissions and improve throughput performance; we do recognize that it may be difficult to actually minimize the number of transmissions in practice.

Finally, note that the probe messages are of small size (128 bytes) and are sent at the basic rate. For the transmission of the actual data, which are much larger (1500 bytes), Samplerate rate control mechanism is used in our cards [3]. Thus, the probes may over-estimate the probability of data packet delivery. However, our contribution is not a new way of computing the link delivery probabilities; instead we rely on a previously used method (the one used in the original work on ETX [9]).

Comparisons: We compare ETOP-based routing with ETXbased routing [9], since it represents a large class of previously proposed metrics. Other metrics use the ETX cost in conjunction with other features (such as multi-rate capabilities [12], or energy consumption [5]) in the final selection of the paths. The ETOP cost could be possibly used in lieu of the ETX cost and this will be considered in future work. 


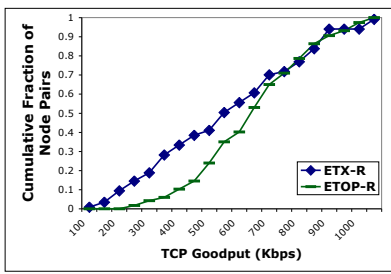

(a) CDF of the TCP goodput for all 110 paths. (b) Median TCP goodput for al path lengths.

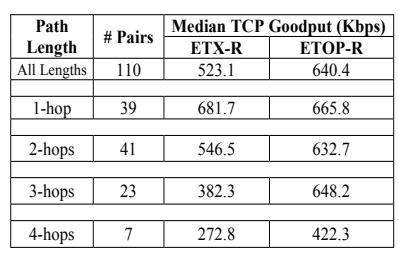

Fig. 3. ETOP-R offers significant improvement, by over 50\%, over ETX-R for the node pairs separated by 3 or more hops.

\section{Experimental Evaluation of ETOP}

In this section we evaluate the performance of ETOP-R and compare it with that of ETX-R. In summary, we make the following main observations:

- ETOP-R improves the median TCP goodput in our testbed when compared to ETX-R. The improvements are more pronounced on longer paths; for three and four hop paths we observe goodput improvements of over $50 \%$.

- The use of ETOP-R reduces the number of link layer transmissions (including retransmissions) significantly compared to ETX-R; for some TCP flows, the reduction is as high as $60 \%$.

- The use of ETOP-R allows TCP to operate at higher congestion windows compared to ETX-R; for a typical flow, we observe that the median window size is about four times higher.

\section{A. Impact of ETOP-R and ETX-R on long lived TCP Flows}

The Set up: For the first set of experiments, we choose at random a large number of source-destination pairs, 110, out of the possible $25 \times 24=600$ possible combinations and run TCP sessions on each pair for 3 minutes. The time used is similar to that in the experiments reported in [11]. We use "Iperf" [2] to measure the maximum achievable TCP bandwidth (goodput). To make the results between the metrics comparable, the following setup (similar to that in [9]) is used. For each of the 110 node pairs, we run ETX-R immediately followed by ETOP-R. Thus, the results with the two metrics are obtained within minutes of each other; we expect the channel conditions to have changed little during this time ${ }^{5}$. On every path, the protocols are allowed to run for 90 secs to achieve stable operations. Then, the source pings the destination for $5 \mathrm{sec}$, at a rate of one packet per second, to allow the protocols to discover the paths to the destination. The source then initiates a TCP connection with the destination. Every run (for the 110 pairs) takes approximately 15 hours. We repeat the experiment six times and compute an average to reduce the impact of temporal variations.

ETOP-R improves TCP goodput over ETX-R: In Figure 3 the $\mathrm{CDF}$ and the median ${ }^{6}$ of the distribution of the measured TCP goodputs for ETOP-R and ETX-R are depicted. The CDF shows that ETOP-R performs better than ETX-R, by over

\footnotetext{
${ }^{5}$ While this holds in most cases, it is not always true.

${ }^{6}$ When the distribution of the data is skewed (as it is in our case), the median is more representative of a typical observation than the mean [14].
}

\begin{tabular}{|l|c|c|c|c|c|c|c|c|c|c|c|}
\hline & \multicolumn{2}{|c|}{$\mathbf{1 3 - > 2 4}$} & \multicolumn{2}{c|}{$\mathbf{1 9 - > 1 6}$} & \multicolumn{2}{c|}{$\mathbf{2 0 - > 2 4}$} & \multicolumn{2}{|c|}{ 28->18 } & \multicolumn{2}{|c|}{ 28->13 } \\
\cline { 2 - 12 } & ETX-R & ETOP-R & ETX-R & ETOP-R & ETX-R & ETO-R & ETX-R & ETOP-R & ETX-R & ETOP-R \\
\hline Goodput (Kbps) & 0 & 309 & 372 & 289 & 188 & 325 & 312 & 303 & 88.8 & 342 \\
\hline MAC EnR & 15.3 & 10.3 & 6 & 7.2 & 16.2 & 9.6 & 11.4 & 10.5 & 12.5 & 8.8 \\
\hline MAC EnD (\%) & 83.3 & 3.8 & 1.5 & 3.6 & 13.4 & 3.9 & 4.3 & 3.4 & 9.7 & 2.8 \\
\hline W.P. Reliability & 0.41 & 0.78 & 0.78 & 0.86 & 0.56 & 0.76 & 0.64 & 0.82 & 0.72 & 0.76 \\
\hline W.P. Length & 3 & 4.4 & 3 & 3.1 & 3.9 & 4.4 & 3.5 & 4.7 & 3.3 & 4.3 \\
\hline
\end{tabular}

Fig. 4. ETOP-R reduces the link layer retransmissions and packet drops, which leads to higher TCP goodput.

$50 \%$, in a wide range of goodputs. The improvement in the median throughput over all the 110 pairs is around $23 \%$ and surpasses $50 \%$ for those pairs separated by 3 or more hops. Sorting the CDF by path length (depicted in our preliminary work in [15] and omitted here for brevity), reveals that the goodput values where ETOP-R offers significant improvement correspond to those achieved by node-pairs separated by three or more hops; the goodput values where ETOP-R and ETX$\mathrm{R}$ perform statistically the same correspond to those achieved by node-pairs one or two hops away. This is expected, since, for the node pairs that are separated by one or two hops the position of the link has little or no relevance and thus, ETOP-R can offer little or no improvements.

\section{B. MAC level performance of ETOP-R and ETX-R}

ETOP-R is designed to reduce the retransmissions costs at the MAC layer and thus, it is important that we examine whether ETOP-R has met its design goals. To this end, we focus our attention on 5 node pairs for which the paths were among the longest in our testbed; as discussed earlier both ETX-R and ETOP-R are designed to provide improvements over long paths. Iperf is once again used to establish long-lived TCP flows; only 1 flow is active at a time. During runtime, using the athstats tool provided with the MadWifi driver [3], for each flow, at every node that participated in that flow, the following statistics are gathered at the MAC layer: (i) the number of transmissions (including retransmissions), (ii) the number of packets that were dropped after the maximum number of retransmissions was exceeded. In our experiments we disable the RTS/CTS, thus, the default long retry limit ${ }^{7}$ of 7 is used by the wireless cards.

To present the data in a meaningful way, we define two new measures: the Effective Number of transmissions and Retransmissions (EnR), and the Effective number of Dropped packets (EnD). For a TCP flow, the EnR is calculated as the ratio of the packets received at the destination to the total number of transmissions and retransmissions attempted at the MAC layer while this TCP flow was alive. This measure is a good estimate of the cost of delivering a TCP packet successfully from the source to the destination. Similarly, the EnD for a TCP flow is calculated as the ratio of all the packet drops to the number of transmissions (retransmissions are not counted) performed by the MAC of the source, while the specific TCP flow was alive. This measure is a good estimate of the percentage of the

\footnotetext{
${ }^{7}$ We intended to perform experiments with varying retry limits. However, in our cards this functionality is implemented in the firmware, to which we could not obtain access.
} 
packets that were sent by the MAC of the source and were dropped somewhere along the path. In addition to these two measures, in Figure 4, we show the TCP goodput, the weighted path reliability and length, for all the five TCP flows. We record all the paths traversed by a TCP flow during the experiment and the number of packets sent over each of the paths. The reliability (the product of the delivery probabilities of the links on the path) and length of each path are weighted by the number of packets sent over that path to compute the latter two metrics.

The use of ETOP-R reduces MAC transmissions and packet drops: Our experiments validate our intuition that ETOP-R reduces the number of transmissions needed for e2e reliable data delivery. As seen in Figure 4, ETOP-R typically reduces he EnR significantly, which in turn leads to higher TCP goodput. Furthermore, we notice that ETOP-R generates more reliable (which leads to reduced $E n D$ ) but longer paths compared to ETX-R. This is a direct consequence of the ETOP-R design. ETOP-R assigns higher costs to the packet drops close to the destination (See Equation (7), in Section IV) and thus, it prefers more reliable although possibly longer alternatives.

Examining the paths computed by ETOP-R and ETX-R Next, we examine the actual routes that were computed with ETX-R and ETOP-R (during the process of collecting the data depicted in Figure 4) for 3 of the 5 node pairs, $13 \rightarrow 24,19 \rightarrow 16$, $20 \rightarrow 24$ to obtain a better understanding of the results reported so far. The first two pairs were chosen because the TCP goodput achieved in the two cases considerably deviates from what was typically observed in Figure 3. In particular, with ETX-R, Iperf reports a zero goodput for pair $13 \rightarrow 24$; for the pair $19 \rightarrow 16$, ETX-R yields a higher TCP goodput than ETOP-R. The third considered node pair $20 \rightarrow 24$ has a behavior that is typical of most connections of that length i.e., ETOP-R increases the TCP goodput by about $50 \%$ compared to ETX-R.

We depict the paths ${ }^{8}$ and the link success probabilities for three node pairs in Figure 5; only the forward paths, traversed by the TCP data are shown for clarity. Consider the paths selected by ETX-R and ETOP-R for the pair $13 \rightarrow 24$. The paths follow the exact same links up to node 23; but they diverge beyond this point. ETX-R chooses the direct link from node 23 to 24 , whereas ETOP-R chooses the sub-path $23 \rightarrow 30 \rightarrow 24$. As per the design of ETX-R, the direct link is the right choice; the cost of this link with ETX-R is $1 / 0.62=1.61$, while the path $23 \rightarrow 30 \rightarrow 24$ has a cost 2 . However, if a packet transmission fails after the bounded number of link layer retransmission attempts on link $23 \rightarrow 24$, node 13 will have to retransmit the packet anew and the cost will be significantly higher; unlike ETOP-R, ETX-R fails to account for this effect. A closer inspection at why a zero-goodput was achieved with ETX-R in this case, provided insights on this observation. First, as discussed above, ETX-R, due to its inherent design, chose a path that was more unreliable than that chosen by ETOP-R. Second, when the data was collected with ETX-R, the link $13 \rightarrow 22$ was of worse quality compared to when ETOP-R was

\footnotetext{
${ }^{8}$ Since the paths change during a TCP connection, we consider those paths on which most of the data packets were transmitted.
}

used. This occurred despite that the data with the two metrics is collected only a few minutes apart. In summary, with ETX-R, the TCP SYN messages followed a very unreliable path, partly because of the choice made by ETX-R, and partly because of of an unlucky fluctuation in the quality of the link $13 \rightarrow 22$. A look at how the Linux kernel implements the TCP connection establishment phase revealed that the SYN message is sent by the sender at most 5 times. Thus, if a short but unreliable path is chosen, there is a high likelihood that the TCP SYN message will not get through. In the above case, the path generated with ETX-R was of low reliability and thus, TCP failed to establish a connection.

Next we consider the pair $19 \rightarrow 16$. For this pair, the goodput achieved with ETX-R is higher than that with ETOP$\mathrm{R}$ (Figure 4). We observe in Figure 5 that both metrics yield the exact same path. However, due to link quality fluctuations, when the data for ETOP-R was collected, the link $19 \rightarrow 22$ has a delivery ratio of 0.9 , while the same link had a delivery ratio of 1 when the data for ETX was collected. In other words, ETOP-R was unlucky in this case. This caused the difference in TCP goodput.

Finally, we consider the paths chosen for the pair $20 \rightarrow 24$. To reach node 24 from node 28 , ETX-R chooses $28 \rightarrow 29 \rightarrow 24$ over $28 \rightarrow 26 \rightarrow 40 \rightarrow 24$; the latter was chosen with ETOP-R. The path with ETX-R, has a fairly unreliable link at the last hop; each packet drop on this link induces an e2e retransmission and this results in a significant increase in retransmission load. Indeed in our experiments we observed that, with ETX-R 303 packets were dropped at node 29 , which $\approx 7 \%$ of the packets transmitted by the sender, node 20 . This lead to the lower TCP goodput with ETX-R as compared to ETOP-R.

\section{Effect of ETOP-R and ETX-R on the TCP Congestion Window}

The sender's congestion window roughly represents the amount of data that the sender can inject into the TCP pipe. To collect the sender's congestion window used by TCP we had to insert macros at specific places in the TCP implementation in the Linux kernel. The macros collect the values of the specific parameters and export them into user space. For brevity and clarity, we focus on one of the five node pairs that were considered in the previous set of experiments, namely node pair $20 \rightarrow 24$. Similar behavioral results were observed for other node pairs. We plot the TCP goodput observed during a 5 minute experiment for both ETX-R and ETOP-R in Figure 6. We also show the congestion window over a shorter period during the experiment; the shorter period is chosen for clarity in the plot and the behavior shown is representative of that throughout the experiment. The results show that the congestion window is consistently higher with ETOP-R than with ETX-R. This is a direct artifact of the increased reliability; given that fewer packets are dropped, TCP is able to aggressively ramp up its congestion window. This in turn translates into a significantly higher TCP goodput as observed in Figure 6(a).

\section{Conclusions}

In this paper, we revisit the problem of computing the path with the minimum cost in terms of the number of link 


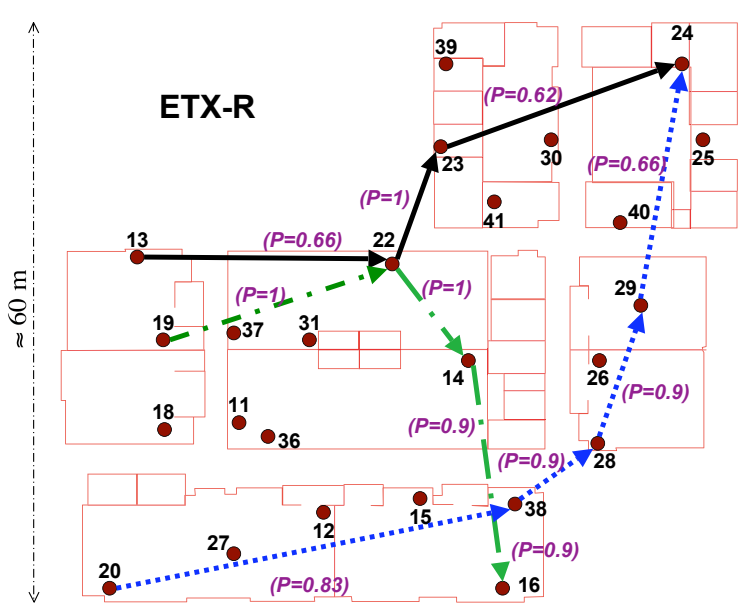

(a) The paths found by ETX-R.

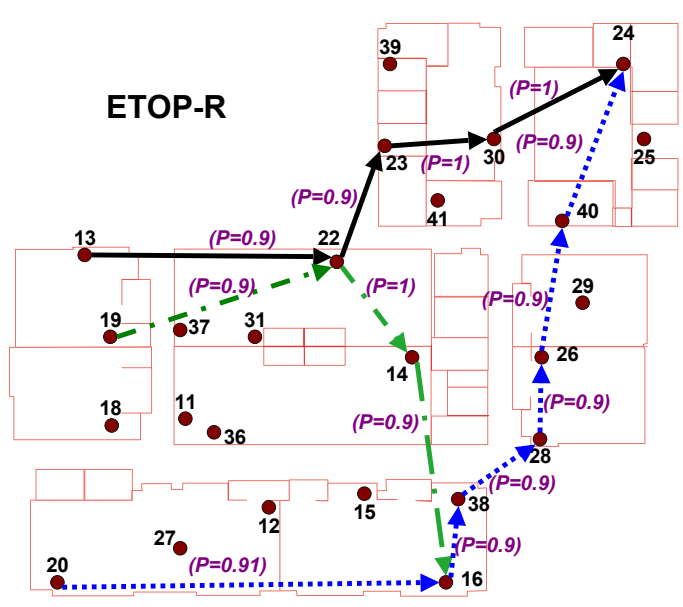

(b) The paths found by ETOP-R.

Fig. 5. The paths and link reliabilities for ETOP-R and ETX-R for three node pairs.

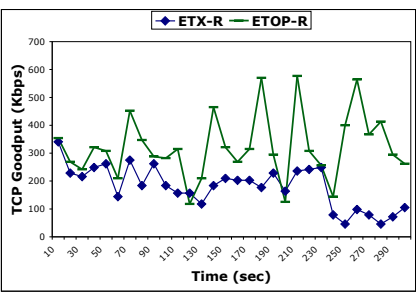

(a) TCP Goodput

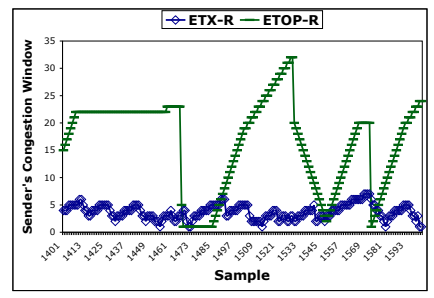

(b) Sender's Congestion Window.
Fig. 6. With ETOP-R, TCP is able to aggressively ramp up its congestion window and achieve a higher goodput.

layer transmissions and retransmissions in multi-hop wireless networks. The key feature that distinguishes our work is that we consider a finite number of link level retransmissions, unlike previous efforts (such as ETX). We demonstrate that in addition to the magnitude of the link reliabilities on a path, the relative ordering of the links is critical in computing the correct minimum cost path. We provide an analytical model to compute a noncommutative path metric, ETOP, that captures this cost. We show that in spite of ETOP's complex form, the problem of computing the path with the minimum ETOP value can be solved using our greedy routing strategy. We implement ETOPbased routing and perform extensive experiments on a 25 node indoor mesh network to quantify and evaluate its performance. We compare the performance of the paths computed with our metric with those computed with a routing strategy based on ETX. Our scheme outperforms the ETX based routing, by over $50 \%$ in many cases, in terms of TCP goodput.

\section{REFERENCES}

[1] Emp-8602 6g wireless card. http://www.netgate.com.

[2] Iperf-tool.http://dast.nlanr.net/projects/iperf/.

[3] The MadWifi driver. http://madwifi.org.

[4] D. Aguayo, J. Bicket, S. Biswas, G. Judd, and R. Morris. Link-level measurements from an 802.11b mesh network. In ACM SIGCOMM 2004.

[5] S. Banerjee and A. Misra. Minimum energy paths for reliable communication in multi-hop wireless networks. In ACM MobiHoc 2002.

[6] C. Koksal and H. Balakrishnan. Quality-Aware Routing Metrics for TimeVarying Wireless Mesh Networks. IEEE JSAC, 24(11), November 2006.

[7] A. Cerpa, J. L. Wong, M. Potkonjak, and D. Estrin. Temporal properties of low power wireless links: modeling and implications on multi-hop routing. In ACM MobiHoc 2005.
[8] T. Cormen, C.Leiserson, and R.Rivest. Introduction to Algorithms. McGraw Hill, 2000.

[9] D. S. J. De Couto, D. Aguayo, J. Bicket, and R. Morris. A highthroughput path metric for multi-hop wireless routing. In ACM MobiCom 2003.

[10] Q. Dong, S. Banerjee, M. Adler, and A. Misra. Minimum energy reliable paths using unreliable wireless links. In ACM Mobihoc 2005.

[11] R. Draves, J. Padhye, and B. Zill. Comparison of routing metrics for static multi-hop wireless networks. In ACM SIGCOMM 2004.

[12] R. Draves, J. Padhye, and B. Zill. Routing in multi-radio, multi-hop wireless mesh networks. In ACM MobiCom 2004.

[13] D. Ganesan, B. Krishnamachari, A. Woo, D. Culler, D. Estrin, and S. Wicker. Complex behavior at scale: An experimental study of lowpower wireless sensor networks. technical report csd-tr 02-0013, ucla, february 2002.

[14] R. Jain. The Art of Computer Systems Performance Analysis. John Wiley and Sons, Inc., 1991.

[15] Gentian Jakllari, Stephan Eidenbenz, Nick Hengartner, Srikanth V. Krishnamurthy, and Michalis Faloutsos. Extended abstract: Revisiting minimim cost reliable routing in wireless mesh networks. In ACM MobiCom '07.

[16] D. B. Johnson, D. A. Maltz, and Y. Hu. The Dynamic Source Routing Protocol for Mobile Ad Hoc Networks (DSR). Internet draft (work in progress). IETF, April 2003.

[17] J.Proakis. Digital Communications. McGraw Hill, 2000.

[18] K. Kim and K. G. Shin. On accurate measurement of link quality in multi-hop wireless mesh networks. In ACM MobiCom '06.

[19] E. Kohler, R. Morris, B. Chen, J. Jannotti, and M. F. Kaashoek. The click modular router. ACM Trans. Comput. Syst., 18(3):263-297, 2000.

[20] D. Kotz, C. Newport, R. S. Gray, J. Liu, Y. Yuan, and C. Elliott. Experimental evaluation of wireless simulation assumptions. In $A C M$ MSWiM 2004.

[21] X. Li, Y. Shu, H. Chen, and X. Chu. Energy efficient routing with unreliable links in wireless networks. In IEEE MASS 2006.

[22] H. Lundgren, E. Nordstro, and Ch. Tschudin. Coping with communication gray zones in ieee $802.11 \mathrm{~b}$ based ad hoc networks. In ACM WOWMOM 02 .

[23] K. Seada, M. Zuniga, A. Helmy, and B. Krishnamachari. Energy-efficient forwarding strategies for geographic routing in lossy wireless sensor networks. In ACM SenSys '04.

[24] A. Woo, T.Tong, and D. Culler. Taming the underlying challenges of reliable multihop routing in sensor networks. In ACM SenSys '03.

[25] M. D. Yarvis, W. S. Conner, L. Krishnamurthy, A. Mainwaring, J. Chhabra, and B. Elliott. Real-world experiences with an interactive ad hoc sensor network. In IEEE ICPPW 2002.

[26] H. Zhang, A. Arora, and P. Sinha. Learn on the fly: Data-driven link estimation and routing in sensor network backbones. In IEEE INFOCOM, 2006. 